\title{
Epidemiologia delle parassitosi intestinali in una popolazione di immigrati extracomunitari
}

\author{
Espedito Nocera', Lorella Bozzelli', Maria Assunta Gallo', Mariarosaria Rea ${ }^{2}$, \\ Vincenzo Cuniato 3 , Marinella Di Martino ${ }^{3}$, Renato Natale ${ }^{3}$, Daniele Crotti ${ }^{4}$ \\ 'Centro Diagnostico S. Ciro, Portici (NA). \\ 'Laboratorio Kappa, Pomigliano d'Arco (NA). \\ ${ }^{3}$ Centro medico-sociale J.E.Masslo, Castelvolturno (CE). \\ ${ }^{4}$ Libero Docente in Parassitologia (già Responsabile Sezione di Microbiologia Clinica, Ospedale R. Silvestrini,, Perugia).
}

Key words: Intestinal parasitosis, immigrants, ova \& parasite, epidemiology

\section{Epidemiology of intestinal parasitosis in a population of immigrants}

\section{SUMMARY}

The Authors relate the results of an epidemiological study concerning the percentages of isolation for intestinal parasites in a population of out-community immigrant population that attends the ambulatory of "Jerry Masslo" voluntary Association in Castelvolturno, provence of Caserta, southern Italy. In the period that has gone since November 200 I to January 2003,8 I faecal samples of out-community immigrants were examined by standard coproparassitologyc examination: direct microscopic examination with iodine solution and with physiological solution, microscopic examination after enrichment with formalin-etilacetate and finally microscopic examination after permanent stain of Giemsa. Of all the examined samples, 44 (54.3\%) were positive for one or more intestinal pathogenic parasites, while $37(45.7 \%)$ were negative. The motivations to this study are to seek in the wish to give a contribution, even though least, to the understanding of the sanitary problems that this type of patients, the out-community immigrants, coming to make part in consistent way of our society more and more, has brought with itself.

\section{INTRODUZIONE}

La crescente presenza di immigrati nel nostro paese ha provocato importanti conseguenze sul piano sociale, culturale, economico e soprattutto sanitario (12). In quest'ambito, le malattie a trasmissione sessuale rappresentano sicuramente il problema maggiore per questa categoria di persone (7); pur tuttavia anche le parassitosi, ed in particolare le parassitosi intestinali, costituiscono un capitolo di grande rilevanza microbiologica e, in determinati contesti, infettivologica e di sanità pubblica $(3,5,7,8,12)$. Ciò è conseguenza di svariati fattori: a) provenienza da paesi dove questo tipo di infezioni ed infestazioni sono particolarmente diffuse; b) mancanza di adeguate informazioni sanitarie nel paese d'origine; c) condizione di precarietà sociale ed economica; d) scarsa attenzione da parte delle nostre strutture diagnostiche a queste malattie, soprattutto se asintomatiche o paucisintomatiche; e) non di rado scadente $\mathrm{o}$ insufficiente capacità diagnostica al riguardo da parte di non pochi laboratori biomedici; f) sottostima del fenomeno in quanto non remunerativo nell'attuale panorama sanitario nazionale $(3,5,7$, $8,10,12,13)$.

Scopo del presente studio è stato quello di valuta- re le prevalenze di positività per parassiti intestinali in una sia pur limitata popolazione di immigrati extracomunitari presenti nel cosiddetto "litorale domitio", che è rappresentato da un tratto di costa ad ovest di Napoli, in provincia di Caserta. Le motivazioni a tale ed iniziale studio parassitologico (peraltro inserito in uno ben più ampio, coinvolgente la tutela della popolazione immigrata, soprattutto femminile, sia da un punto di vista medico che sociale) sono scaturite dalla necessità di comprendere appieno le problematiche sanitarie della popolazione afferente ad un Centro Medico gestito volontariamente e gratuitamente da sanitari impegnati da tempo anche nella prevenzione delle patologie di importazione, dalla volontà a qualificare l'impostazione diagnostica proprio in tema di parassitosi intestinali (e quindi di diagnostica coproparassitologica adeguata), dalla coscienza e dalla convinzione della importanza, in ogni senso intesa, di allargare e migliorare la quantità e la qualità delle prestazioni analitiche $(2,3,4,6,13)$.

\section{MATERIALI E METODI}

Nel periodo di tempo che va da Novembre 2001 a Gennaio 2003 sono stati sottoposti ad esame 
copro-parassitologico, presso l'ambulatorio dell'Associazione di volontariato "Jerry E. Masslo" di Castelvolturno (CE), 81 campioni di feci di pazienti extracomunitari di cui 48 donne $(59.3 \%)$ e 33 uomini (40.7\%), con un'età media di 24 anni. Per ogni paziente è stata compilata una scheda anamnestica in cui sono stati registrati oltre ai dati sanitari, anche i dati socio-demografici e comportamentali. Il maggior numero di immigrati era proveniente dalla Nigeria e dal Ghana, mentre i rimanenti erano originari di altre nazioni africane e, in piccola percentuale, di Paesi dell'Europa orientale (figura I).

Va precisato che tale indagine voleva avere una valenza esclusivamente epidemiologico-conoscitiva, in quanto tutti i soggetti afferivano all'ambulatorio dell'Associazione per altre patologie, principalmente relative alla sfera genitale. Pertanto, nella popolazione analizzata, non è stato condotto alcun tipo di stratificazione finalizzato ad eventuali correlazioni tra sintomatologia intestinale, area geografica di provenienza e parassitosi enteriche rilevate $(3,4)$.

Per ogni campione fecale veniva eseguito inizialmente un esame macroscopico per escludere la presenza di parassiti macroscopicamente rilevabili (proglottidi di tenie o eventuali adulti di ossiuri). Quindi veniva effettuato l'esame coproparassitologico standard (O \& P): esame microscopico diretto, esame microscopico dopo arricchimento formolo-etilacetato ed esame microscopico dopo colorazione permanente di Giemsa $(2,13)$. Le osservazioni microscopiche erano condotte sia in soluzione fisiologica che in soluzione iodata secondo Lugol. Per la colorazione permanente, i preparati, fissati con metanolo ed asciugati all'aria, venivano ricoperti da una soluzione di Giemsa al 3\% ivi mantenuta per un'ora; una volta asciugati, i vetrini venivano osservati al microscopio in immersione per l'identificazione dei trofozoiti, in particolare dei flagellati patogeni (1, $13)$.

Ad ogni soggetto venne eseguito l'O \& P soltanto su un solo campione fecale, in quanto trattatasi di una indagine epidemiologica conoscitiva $(3,4)$. Esula dallo studio, inoltre, sia la ricerca mirata di ossiuri (Enterobius spp.), per la ricerca delle cui uova è necessario ricorrere allo scotch-test, sia la ricerca mirata di Strongyloides stercoralis, sia la ricerca di Cryptosporidum spp., per la quale è necessario utilizzare una colorazione all'acidoresistenza $(1,2,13)$. Tale scelta fu anche giustificata dal fatto che trattavasi di soggetti adulti, senza disturbi specifici in sede anale e senza diarrea in atto, oltre al fatto, si ribadisce, del significato puramente conoscitivo che l'indagine voleva avere $(1,2,3,4)$.
Nei dettagli va detto che la programmazione dello studio parassitologico fu la seguente. Le osservazioni macroscopiche e le osservazioni microscopiche dirette venivano eseguite presso il Centro Diagnostico S. Ciro, che fungeva da centro coordinatore; le osservazioni microscopiche dopo arricchimento venivano eseguite in triplo presso il Centro S. Ciro, il Laboratorio Kappa di Pomigliano d'Arco ed il Centro di Riferimento di Perugia; le osservazioni microscopiche dopo colorazione di Giemsa (eseguita sempre presso il Centro S. Ciro) venivano eseguite presso il Centro di Riferimento di Perugia. Tutti i casi discrepanti, conservati in Lugol e in formalina, venivano inviati, rivisti e riverificati tutti dal Centro di Riferimento di Perugia. Mentre i campioni di volta in volta raccolti pervenivano ai due laboratori campani entro 12-24 ore, i medesimi campioni, una volta conservati, venivano spediti a Perugia ogni 3-4 mesi.

\section{RISULTATI}

I risultati, in frequenza, percentuale e prevalenza, sono riportati in tabella 1.

Sugli 81 soggetti esaminati, 44, pari al $54.3 \%$, sono risultati essere parassitati da uno o più parassiti patogeni, mentre 37 soggetti, pari al $45.7 \%$, sono risultati negativi per qualsivoglia parassita indagato $(3,10)$.

Nel complesso la frequenza di reperimento dei protozoi è stata del $27.2 \%$ (22 casi). Tra questi, Dientamoeba fragilis (figura II) ha nettamente prevalso, essendo stata riscontrata nel 23.5\% (19 casi), mentre assai inferiori sono state le percentuali degli altri protozoi patogeni (o potenzialmente tali): Entamoeba histolytica/dispar, con il $2.5 \%$ ( 2 casi) e Giardia duodenalis con 1'1.2\% (1 caso).

La frequenza totale delle elmintiasi è stata anch'essa del $27.2 \%$, sempre pari a 22 casi; tra questi nettamente prevalenti sono apparsi essere gli ancilostomidi (figura III) con 17 casi, pari al 21.1\%; seguono Trichuris trichiura (figura IV) con il 3.7\% (3 casi), Schistosoma mansoni con $1 ' 1.2 \%$ (1 caso), e Ascaris lumbricoides (figura V) anch'esso con 1' $1.2 \%$ (1 caso).

Va però ed inoltre precisato che, in circa il $44 \%$ dei soggetti, sono stati evidenziati, da soli o in associazione tra loro o con patogeni, vari protozoi commensali. Quest'ultima, ed in fondo elevata, percentuale, viene qui segnalata sia per dovere di completezza diagnostica sia perché, in ogni caso, è o può essere espressione di una contaminazione fecale in primis ambientale, e quindi da segnalare, in ogni caso $(1,3,4,13)$. Sono state così individuate essenzialmente cisti di amebe non patogene quali Entamoeba coli, Endolimax nana, 
Iodamoeba buetschlii e, soprattutto, cisti di Blastocystis hominis $(1,2,6)$.

Per quanto riguarda la correlazione tra enteroparassiti isolati e sintomatologia, abbiamo osservato che dei 44 pazienti in cui è stata evidenziata la presenza di parassiti patogeni, solo 5, pari al 14.7 $\%$, presentavano qualche segnale di interessamento intestinale, rappresentato per lo più da dolori addominali e diarrea saltuaria. Ma è altresì evidente come, nonostante il colloquio preliminare con i singoli soggetti, non tutte le informazioni, per vari motivi (non ultima la lingua), sono state raccolte, comprese o fornite. Ma questo non diminuisce il nostro piccolo studio, che dovrebbe essere, per anticipare la brevissima discussione, preliminare per proseguire con indagini ben più razionali, mirate e programmate, sulla base anche e proprio da quanto emerso da questa iniziale indagine.

Tabella I. Distribuzione delle positività per parassiti patogeni PARASSITI NUMERO SOGGETTI PERCENTUALE

\begin{tabular}{lll}
\hline Dientamoeba fragilis & 19 & $23.5 \%$ \\
\hline Ancilostomidi & 17 & $21.1 \%$ \\
\hline Trichuris trichiura & 3 & $3.7 \%$ \\
\hline Entamoeba histolytica & 2 & $2.5 \%$ \\
\hline Giardia duodenalis & $\mathrm{I}$ & $1.2 \%$ \\
\hline Schistosoma mansoni & $\mathrm{I}$ & $1.2 \%$ \\
\hline Ascaris lumbricoides & $\mathrm{I}$ & $1.2 \%$ \\
\hline
\end{tabular}

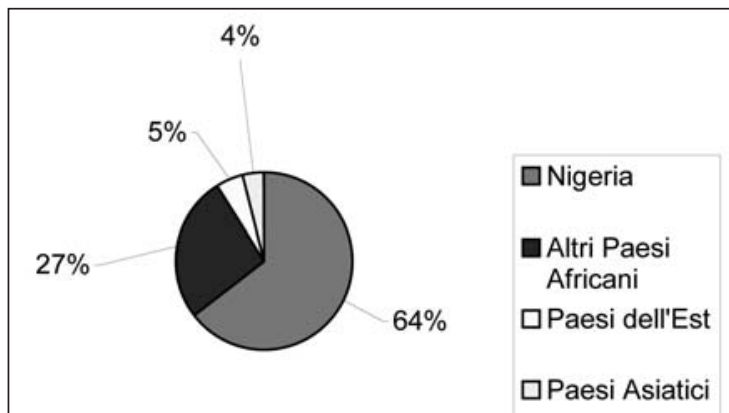

Figura I. Grafico relativo ai paesi di origine degli extracomunitari

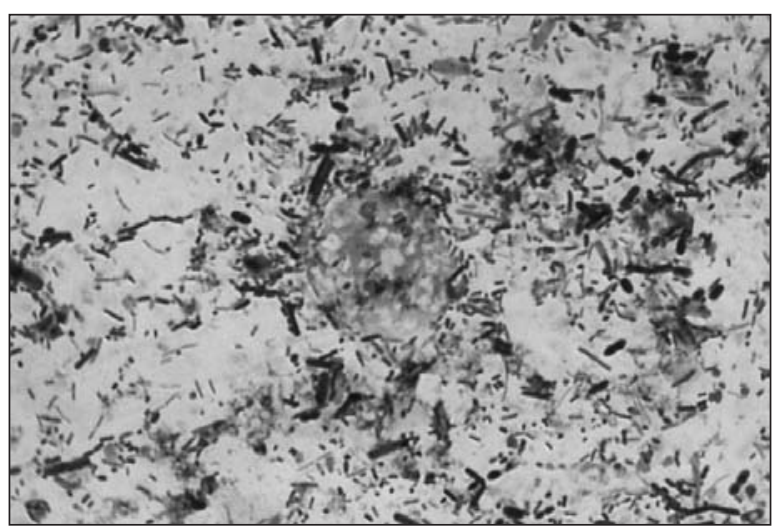

Figura II. Trofozoite di Dientamoeba fragilis (colorazione di Giemsa)

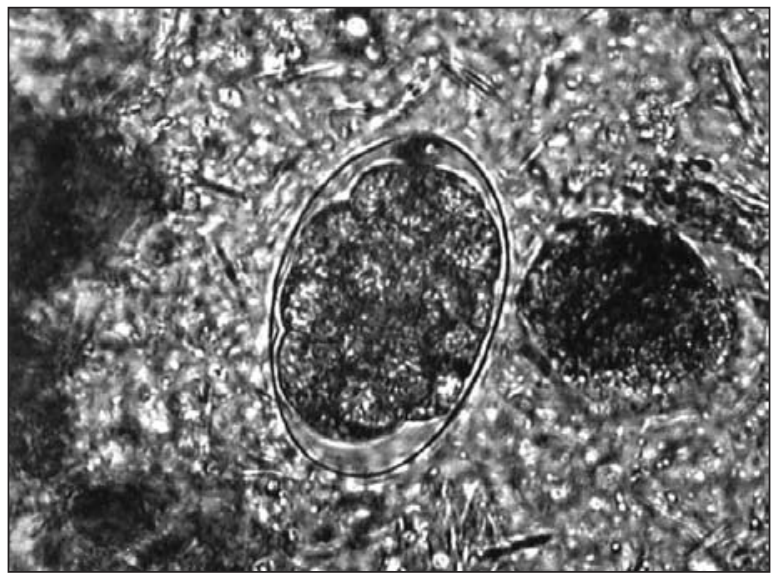

Figura III. Uova di ancilostomide (preparato a fresco in soluzione di Lugol)

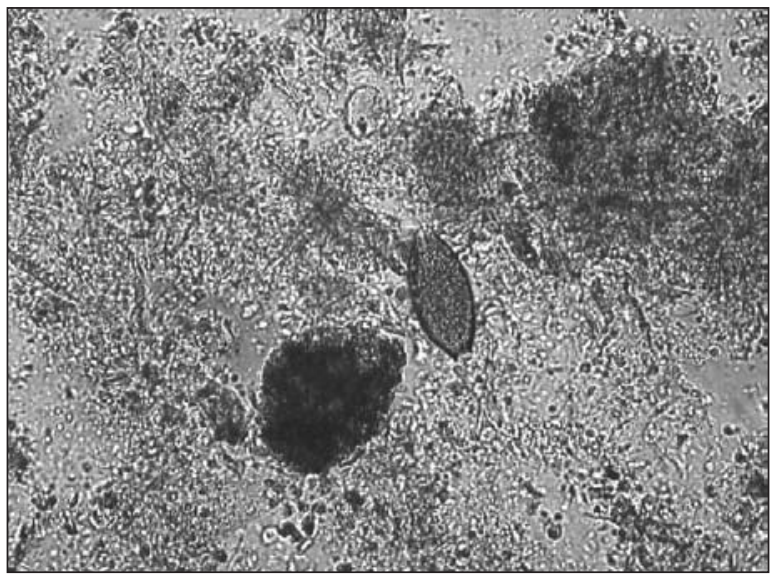

Figura IV. Uovo di Trichuris trichiura (preparato a fresco in soluzione di Lugol)

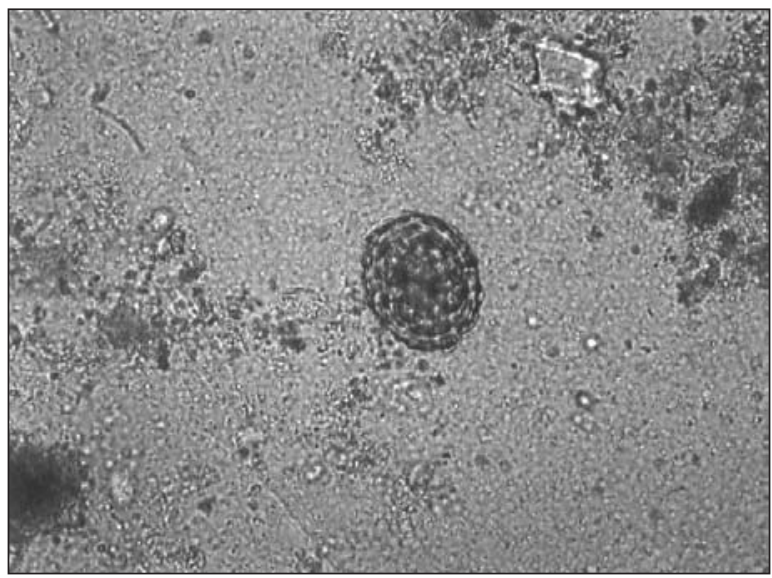

Figura V. Uovo di Ascaris lumbricoides (preparato a fresco in soluzione di Lugol)

\section{DISCUSSIONE}

Come si evince dai dati sopra riportati, ci troviamo di fronte a percentuali di positività ben più elevate rispetto a quelle che caratterizzano gli studi condotti su popolazioni autoctone, anche se si prendono in esame analisi effettuate su gruppi 
di persone socialmente disagiate che più sono rapportabili alla particolare tipologia degli individui reclutati in questo lavoro, ossia extracomunitari spesso in condizione di clandestinità $(3,4,5,7$, 12).

Data l'esiguità della popolazione esaminata, in questa iniziale indagine, e la scarsezza delle informazioni cliniche ricevute (anche in virtù del tipo di approccio avuto) non ci è parso razionale fornire alcune correlazioni statistiche, anche perché superflue e premature, tra parassiti individuati e caratteristiche dei soggetti arruolati.

A questa, che è la più immediata considerazione derivante dai dati sopra riportati, se ne possono aggiungere altre che sono, riteniamo, altrettanto interessanti e, soprattutto, stimolanti per futuri approfondimenti:

a. la maggior parte degli extracomunitari nei quali è stata riscontrata la presenza di enteroparassiti è apparentemente priva di qualsiasi tipo di sintomatologia clinica, il che può far ritenere che questi individui siano in effetti da considerarsi portatori sani (o apparentemente tali) con le conseguenze negative derivanti: nessuna richiesta di intervento medico, nessuna terapia effettuata e quindi potenziale diffusione incontrastata nell'ambiente di parassiti indesiderati;

b. i risultati sopra ottenuti sono ricavati da soggetti che per lo più hanno lasciato da poco il paese d'origine, in genere ad alta endemia parassitaria, per cui è comunque ipotizzabile che trascorso un certo lasso di tempo, ed in seguito a variate condizioni ambientali (si spera migliorate, soprattutto dal punto di vista igienico, rispetto a quelle in cui erano abituati a vivere) buona parte di questi soggetti possa "depurarsi";

c. d'altra parte, v'è purtroppo da considerare, realisticamente, che una percentuale non certo limitata degli individui in esame, per tutta una serie di motivi (sociali, politici, razziali ecc.) che in questa sede non ci sembra il caso di approfondire, continueranno a perpetrare un tenore di vita di "infimo" livello e quindi convivere con quei fattori ambientali (sovraffollamento, scarsezza di igiene, carenze alimentari, mancati controlli medici) che sicuramente tanto hanno contribuito all'acquisizione, al mantenimento ed alla diffusione delle enteroparassitosi in oggetto.

Per quanto riguarda la disamina delle parassitosi osservate, non deve stupire, essenzialmente, la maggior frequenza di $D$. fragilis, rispetto a $G$. duodenalis o ad altri parassiti. D. fragilis, protozoo da tempo sottostimato, è ora tutt'altro che raro e, laddove debitamente ricercato, rappresenta il protozoo e forse il parassita più frequente, quantomeno nella popolazione autoctona $(1,2,9$, 11, 14).

Alla luce di tutto ciò è chiaro ed auspicabile che ben più ampie e diversificate indagini dovranno essere effettuate su un numero maggiore di soggetti extracomunitari e le medesime ripetute ad intervalli di tempo ragionevolmente cadenzati per i motivi sopra accennati. Il tutto per poter inquadrare al meglio l'epidemiologia delle enteroparassitosi diffuse tra gli extracomunitari, che sempre più rapidamente ed in numero sempre più elevato entrano a far parte della nostra società e, perché no, per poter contribuire, seppure in modo indiretto, a migliorare le condizioni di vita di queste persone $(6,9)$.

\section{BIBLIOGRAFIA}

1. Bernieri F, Crotti D. Infezioni da protozoi. Microbiol Med 2001, 16(I): 98-108.

2. Bernieri F, Crotti D, Galli D, Raglio A. Manuale illustrato di diagnostica parassitologia, Selecta Medica Edizioni, Pavia, 2001

3. Bernieri F, Galli D, Crotti D, et al. Studio Policentrico dell'Associazione Microbiologi Clinici Italiani Comitato di Studio di Parassitologia (AMCLI-CoSP) sulla diffusione delle parassitosi intestinali in Italia: parassitosi intestinali nell'immigrato. Giorn It med Trop 1996, 1: 97-99.

4. Bernieri F, Galli D, Giordano S, et al. Indagine nazionale AMCLI-CoSP sulla diffusione delle parassitosi intestinali. Microbiol Med 1994, 9: 415-8.

5. Bevilacqua N, Damiano F, Ricci F, Maresca G. Immigrazione e patologie infettive: dieci anni di esperienza della Clinica di Malattie Infettive dell'Università Cattolica, La Medicina Tropicale nella cooperazione allo sviluppo 1992; 8: 51-3.

6. Chiodini PL, Moody AH, Manser DW. Atlas of Medical Helminthology and protozoology, Churchill Livingstone, Harcourt Publishers Limited, London, IV Edition, 2001.

7. Corachan M. Immigration and health risks. Giorn Mal Inf Parass 1994, 45: 625-8.

8. Crotti D, Chiacchella A, Geranio N. Incidenza di parassitosi intestinale in studenti di colore in Perugina. Biologi Italiani 1990, 3: 41-3.

9. Crotti D, D’Annibale ML, Fonzo G, Lalle M, Cacciò SM, Pozio E. Dientanoeba fragilis is more prevalent than Giardia duodenalis in childen and adults attendine a day care centre in Central Italy. Parasite 2005, 12: $165-70$.

10. Crotti D, Del Sante M, Fonzo G, Gebremariam T. Schistosomiasi e parassitosi intestinali nella popolazione scolare dell'area di Mendeferà, Eritrea. G It Med Trop 1999, 4: 15-9.

11. De Canale E, Tessari A, Campion L, Rossi L. Dientamoeba fragilis: is it really fragilis? Approach to specimen handling and rapid microscopic diagnosis. Parassitologia 2003, 45: 19-22.

12. Favaro G, Tognetti Bordogna M. La salute degli immigrati. Edizioni Nicopoli, Milano, 1990.

13. Garcia LS. Diagnostic Medical Parasitology. ASM Press, Whashington DC, IV Edition 2001. 
14. Johnson EH, Windsor JJ, Clark CG. Emerging from obscurity: biological, clinical and diagnostic aspects of Dientamoeba fragilis. Clin Microbiol Rev 2004; 17: 553-70.

\section{Espedito Nocera}

Via Savorito 29 - C/mare di Stabia (NA)

Tel. 081 7751033; Fax 0817762863

E-mail: espeditonocera@virgilio.it 